\title{
Simple and reliable model for estimation of methanol cross-over in direct methanol fuel cells and its application on methanol-concentration control
}

\author{
Federico Zenith $^{a b}$ and Ulrike Krewer ${ }^{* a b}$ \\ Received 7th September 2010, Accepted 4th November 2010 \\ DOI: 10.1039/c0ee00415d
}

A simplified model of mass-transport phenomena on the anodic side of direct methanol fuel cells (DMFCs) is presented, with the objective of estimating the cross-over flux in order to enable feedforward (sensorless) control of anodic concentration in DMFC systems. The effect of parameter uncertainty on the tracking error of the control system is analysed and several models for temperature dependence are proposed. Experimental data on methanol cross-over was gathered in a DMFC system, and the models were discriminated by means of nonlinear regression. The regression results and an initial test run indicate that feedforward control of anodic methanol concentration in DMFC systems is feasible.

\section{Introduction}

Direct methanol fuel cells (DMFCs) have a significant potential as portable power sources. Their fuel, methanol, remains in liquid form in any climatic condition ever measured on the planet, ${ }^{\dagger}$ and has a theoretical energy density that dwarfs modern lithium-ion batteries. ${ }^{*}$

This combination of ease of portability and high energy density allows envisioning new possibilities in portable power, dramatically increasing the autonomy range of present portable devices and possibly spurring the invention of new devices whose power requirements cannot be satisfied by today's portable power sources.

However, it is also known that DMFCs have a poor overall conversion efficiency: values in the range of $20 \%$ to $30 \%$ from methanol's stored Gibbs free energy to electrical power are typical,,$^{3,16}$ and a significant fraction of methanol (depending on operating condition) is lost to cross-over flux, a parasitic loss of methanol.

In our previous work ${ }^{18}$ it was theoretically demonstrated what had been empirically noticed by $\mathrm{Ha}$ et al. ${ }^{5}$ i.e. that cross-over stabilises the dynamics of methanol concentration and allows the usage of a purely feedforward control strategy.

Purely feedforward concentration control is desirable because of the difficulty with which methanol concentration in water can be measured: concentration measurements are unreliable, cumbersome or expensive. ${ }^{19}$ Feedforward control does not require a measurement on methanol concentration, and allows the system to be more reliable, lightweight and cheaper.

\footnotetext{
${ }^{a}$ Max Planck Institute for Dynamics of Complex Technical Systems, Sandtorstraße 1, 39106 Magdeburg, Germany. E-mail: krewer@ mpi-magdeburg.mpg.de; Fax: +49 3916110 536; Tel: +49 3916110443

${ }^{b}$ Portable Energy Systems, Chair for Process Systems Engineering, Otto von Guericke University, Universitätsplatz 2, 39106 Magdeburg, Germany $\dagger$ The highest and lowest temperatures ever recorded are $331 \mathrm{~K}$ and 184 $\mathrm{K},{ }^{9}$ whereas methanol boils at $338 \mathrm{~K}$ and freezes at $176 \mathrm{~K} .{ }^{10}$

\$ Comparing data from Tarascon and Armand $^{14}$ and thermodynamical data for methanol combustion, ${ }^{10}$ energy density by mass of methanol is $30-40$ times that of Li-ion batteries.
}

The objective of this article is to demonstrate that it is feasible to estimate cross-over to a precision allowing control of methanol concentration with a feedforward strategy. The article seeks also to quantify the reliability of such an estimation.

\subsection{Literature review}

Methanol cross-over in Nafion membranes was first modelled by Verbrugge, ${ }^{15}$ who produced a dynamic first-principle model, which did not include the effect of mass transport to the catalyst layer. The model was compared to experimental data and two parameters were estimated by regression.

The effect of pressure, concentration, temperature and current on methanol permeation was included by Cruickshank and Scott. ${ }^{1}$ Their analysis does not include modelling of diffusion between the anodic bulk and the catalyst layer, even though the inclusion of methanol electro-osmotic drag does introduce a linear dependence of methanol cross-over on current.

Sundmacher et al. $^{13}$ introduced an estimation of cross-over based on a catalyst-layer methanol concentration, using a masstransport coefficient to calculate the difference between bulk and catalyst-layer concentration. The cross-over itself was estimated with an expression involving the Péclet number, which required the calculation of convective flow velocity through the membrane and in turn the determination of several other parameters.

A very detailed model in regard to mass transport was presented by Schultz and Sundmacher, ${ }^{11}$ in which Stefan-Maxwell equations for multicomponent diffusion were employed over seven diffusion compartments (bulk, diffusion layer and catalyst layer of anode and cathode respectively, and the membrane). The diffusion coefficients varied with temperature according to an Arrhenius expression.

Wang ${ }^{17}$ proposed a much simpler lumped model, with crossover flow starting from a nominal value at open circuit and decreasing linearly with increasing current, reaching zero at the cell's mass-transport limit.

In the entire body of literature about cross-over estimation, no publication has yet provided the standard deviations with which all the parameters necessary for cross-over estimation are known, 
nor compared models from the point of view of parameter regression.

\section{Modelling of methanol cross-over}

Cross-over is the phenomenon of methanol diffusion through the membrane separating the anodic and cathodic compartment. Most membranes, such as DuPont's Nafion, rely on water humidification to guarantee proton conductivity. As methanol is infinitely soluble in water, it is inherently difficult to reduce or eliminate cross-over through such a membrane.

Mass transport in DMFCs is a particularly complex topic: in particular, the anodic side features two-phase fluid flow through a porous medium in which capillary effects are significant. ${ }^{20}$

This article, however, proposes a much simpler model structure, illustrated by Fig. 1. It is assumed that there is a single bulk concentration $c$ in the anode compartment and a single catalystlayer concentration $c_{c l} \S$ The mass transport of methanol from the bulk to the catalyst layer is proportional to the mass-transport coefficient $k_{m}$ :

$$
N_{m}=k_{m}\left(c-c_{c l}\right)
$$

Similarly, the cross-over is modelled assuming that the methanol concentration on the cathodic side of the membrane is zero: this is because it is assumed that methanol, upon contact with oxygen on the cathodic catalyst layer, will immediately and completely react. The expression for the cross-over flux is then:

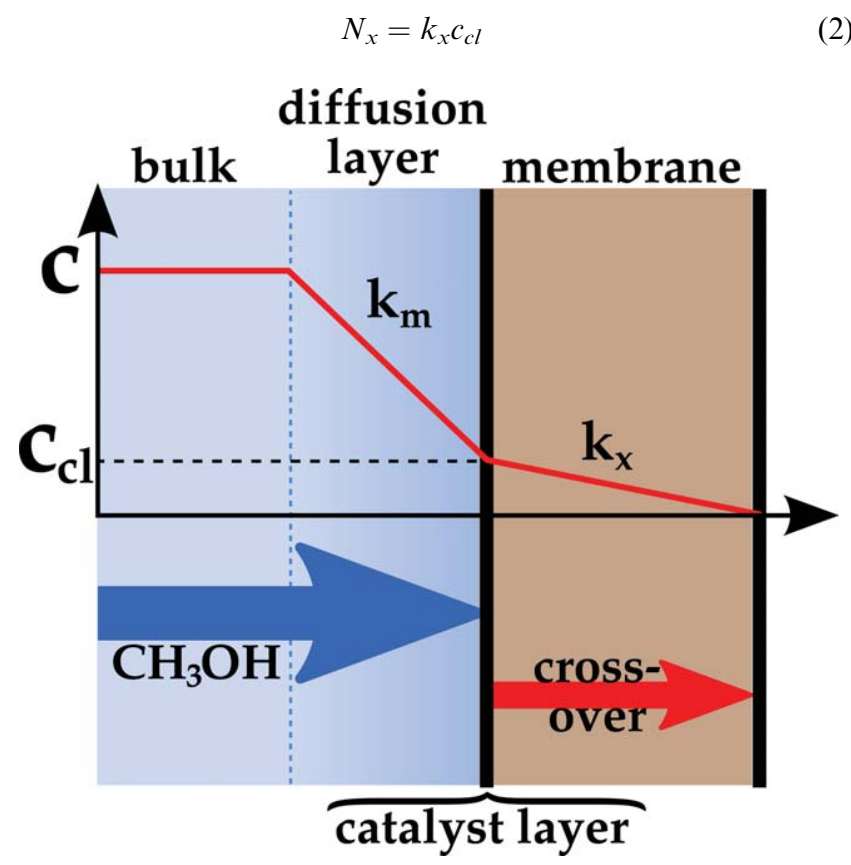

Fig. 1 Methanol concentration profile over the anodic diffusion layer and the membrane.

$\S$ Bulk concentration is in general not uniform over the cell's flow fields, especially in the case of low anodic flow. In that case it would be possible to integrate the methanol consumption over the flow field's length; however, for most practical purposes, constant concentration is an acceptable approximation.
The methanol flux to the catalyst layer $N_{m}$ is composed of the cross-over flux and of the flux due to anodic methanol consumption, which is given by the overall anodic reaction:

$$
\mathrm{CH}_{3} \mathrm{OH}+\mathrm{H}_{2} \mathrm{O} \stackrel{\mathrm{Pt}, \mathrm{Ru}}{\longrightarrow} 6 \mathrm{H}^{+}+6 e^{-}+\mathrm{CO}_{2} \uparrow
$$

Therefore it holds:

$$
N_{m}=N_{x}+\frac{i}{6 F}
$$

Catalyst-layer methanol concentration $c_{c l}$ cannot be measured directly, but can be expressed by rearranging eqns (1), (2) and (4) as:

$$
c_{c l}=\frac{k_{m} c-\frac{i}{6 F}}{k_{m}+k_{x}}
$$

It is then finally possible to express the total methanol flux as:

$$
\begin{aligned}
N_{m} & =\overbrace{\frac{k_{m}}{1+k_{m} / k_{x}}}^{a} c+\left(1-\frac{\overbrace{\frac{1}{1+k_{m} / k_{x}}}^{b}}{6 F}\right) \frac{i}{6} \\
& =a c+(1-b) \frac{i}{6 F}
\end{aligned}
$$

which is notably a function linear in $c$ and $i$; the linearity in current was previously noted by Wang. ${ }^{17}$ Parameters $a$ and $b$ are an equivalent way to represent $k_{m}$ and $k_{x}$, and there is a one-toone correspondence between the two sets of parameters.

Methanol consumption can therefore be described as a function of two variables, methanol concentration and current density, requiring the estimation of two parameters, $k_{m}$ and $k_{x}$ (or, equivalently, $a$ and $b$ ).

\subsection{Effect of parameter uncertainty}

Mass-transport coefficients $k_{m}$ and $k_{x}$ will naturally be known only with a certain precision; it is of obvious interest to find out how much the imprecision in their estimation will propagate to imprecision in the steady-state value of methanol concentration.

At steady state, the methanol feed will be equal to the consumption of methanol in the cell:

$$
\dot{n}_{\mathrm{CH}_{3} \mathrm{OH}}=a A c+(1-b) \frac{I}{6 F}
$$

in which we neglected any further loss terms, which were previously shown to be small. ${ }^{18}$

The control rule to determine $\mathrm{CH}_{3} \mathrm{OH}$ will then be a very similar equation:

$$
\dot{n}_{\mathrm{CH}_{3} \mathrm{OH}}=\hat{a} A \bar{c}+(1-\hat{b}) \frac{I}{6 F}
$$

where the variables with a hat are estimates of the real values, and $\bar{c}$ is the set point for methanol concentration.

Combining eqns (7) and (8), and remembering that $a=k_{m} b$, the control error can be expressed as:

$$
c-\bar{c}=\left(\frac{\hat{a}-a}{a}\right) \bar{c}-\left(\frac{\hat{b}-b}{b}\right) \overbrace{\frac{1}{k_{m}} \frac{i}{6 F}}^{\Delta c_{r}}
$$

$\Delta c_{r}$ is the component of the drop in methanol concentration from anodic bulk to catalyst layer that is due to the anodic reaction 
only. $\Delta c_{r}$ is proportional to $i$, and has a maximum as $i$ approaches the mass-transport barrier value, $i_{\max }$, from which the operating point of a DMFC is usually far. If the control error is not too large, it can be assumed that $\Delta c_{r}<\bar{c}$.

The variance of the control error can be calculated, according to error-propagation theory, as:

$$
\begin{aligned}
\sigma_{c-\bar{c}}^{2}= & \left(\frac{\partial c}{\partial \hat{k}_{m}}\right)^{2} \sigma_{\hat{k}_{m}}^{2}+\left(\frac{\partial c}{\partial \hat{k}_{x}}\right)^{2} \sigma_{\hat{k}_{x}}^{2} \\
& +2\left(\frac{\partial c}{\partial \hat{k}_{m}} \frac{\partial c}{\partial \hat{k}_{x}}\right) \operatorname{Cov}_{\hat{k}_{m}, \hat{k}_{x}}
\end{aligned}
$$

The partial derivatives with respect to estimated parameters $\hat{k}_{m}$ and $\hat{k}_{x}$ are:

$$
\frac{\partial c}{\partial \hat{k}_{i}}=\frac{\bar{c}}{a} \frac{\partial \hat{a}}{\partial \hat{k}_{i}}-\frac{\Delta c_{r}}{b} \frac{\partial \hat{b}}{\partial \hat{k_{i}}}
$$

where $i=\{m, x\}$. The partial derivatives of $\hat{a}$ and $\hat{b}$ are in turn:

$$
\begin{array}{cc}
\frac{\partial \hat{a}}{\partial \hat{k}_{m}}=\frac{\hat{a}}{\hat{k}_{m}}-\frac{\hat{a} \hat{b}}{\hat{k}_{x}} & \frac{\partial \hat{a}}{\partial \hat{k}_{x}}=\frac{\hat{a}^{2}}{\hat{k}_{x}^{2}} \\
\frac{\partial \hat{b}}{\partial \hat{k}_{m}}=-\frac{\hat{b}^{2}}{\hat{k}_{x}} & \frac{\partial \hat{b}}{\partial \hat{k}_{x}}=\frac{\hat{a} \hat{b}}{\hat{k}_{x}^{2}}
\end{array}
$$

Assuming then that $\hat{a} \approx a$ and $\hat{b} \approx b$, the partial derivatives of the control error can be found as:

$$
\begin{gathered}
\frac{\partial c}{\partial \hat{k}_{m}}=\overbrace{\left(\frac{1}{\hat{k}_{m}}-\frac{\hat{b}}{\hat{k}_{x}}\right)}^{>0}+\frac{\hat{b}}{\hat{k}_{x}} \Delta c_{r} \\
\frac{\partial c}{\partial \hat{k}_{x}}=\frac{\hat{a}}{\hat{k}_{x}^{2}}\left(\bar{c}-\Delta c_{r}\right)
\end{gathered}
$$

These expressions still contain the variable quantity $\Delta c_{r} \propto i$. It is also known that this quantity is limited: $\Delta c_{r} \in[0, \bar{c})$. To obtain an absolute limit for the control error's variance, the following inequalities are useful:

$$
\begin{gathered}
\left(\frac{\partial c}{\partial \hat{k}_{m}}\right)^{2} \leq\left(\frac{\bar{c}}{\hat{k}_{m}}\right)^{2} \\
\left(\frac{\partial c}{\partial \hat{k}_{x}}\right)^{2} \leq\left(\frac{\hat{a}}{\hat{k}_{x}^{2}} \bar{c}\right)^{2}
\end{gathered}
$$

In the case of the mixed product, a lower boundary is also of interest for the case of negative covariance:

$$
0 \leq\left(\frac{\partial c}{\partial \hat{k}_{m}} \frac{\partial c}{\partial \hat{k}_{x}}\right)<\frac{\hat{a}}{\hat{k}_{m} \hat{k}_{x}^{2}} \bar{c}^{2}
$$

\subsection{Models proposed for regression}

The model for methanol cross-over should be able to predict, given bulk concentration and current, the methanol consumption in the system. This consumption is given by eqn (6), which takes parameters $k_{m}$ and $k_{x}$ (alternatively expressed with $a$ and $b$ ).
Mass-transport coefficients are known to vary significantly with temperature, and during usage of a DMFC it is expected that temperature will change sufficiently to justify an investigation of how these parameters change.

The mass-transport coefficients can be assumed to be proportional to diffusion coefficients, whose temperature dependence has often been modelled with Arrhenius expressions. ${ }^{8}$ The general expression, both for $k_{m}$ and $k_{x}$, is therefore assumed to be:

$$
\ln \left(\frac{k}{k_{0}}\right)=\frac{E}{R T}
$$

Four models of increasing complexity are proposed and evaluated in this article:

- $k_{m}$ and $k_{x}$ are constant with temperature $(E=0 \mathrm{~kJ} / \mathrm{mol})$;

- $k_{m}$ and $k_{x}$ vary with temperature with the activation energy given by Kauranen and Skou $(E=11.6 \mathrm{~kJ} / \mathrm{mol})$;

- $k_{m}$ and $k_{x}$ vary with temperature with an activation energy to be determined, so $E$ becomes a third parameter.

- $k_{m}$ and $k_{x}$ vary with temperature with two separate activation energies to be determined, so $E_{m}$ and $E_{x}$ become two additional parameters.

\section{Experimental}

\subsection{Equipment}

The rig to gather experimental data is sketched in Fig. 2.

The system's tubings were Tygon 3350 for all flows that could carry a liquid phase and eventually come into contact with the methanol solution, and A-60-G Norprene for the lines that carried only gases.

To measure temperatures in the system it was chosen to use R/ S-type thermocouples, because their materials would not corrode and release metallic ions if exposed to the methanol solution. To bring the thermocouples into contact with the flows, they were arranged as illustrated in Fig. 3: the thermocouple's wires enter a $\mathrm{T}$ connector through its side port, and their hot junction is placed in the path of the fluid flow. To provide rigidity to the joint as well as sealing, a stub of rigid PTFE tubing was fixed with epoxy glue placed around the final part of the thermocouple wire coating and the T connector's side port.

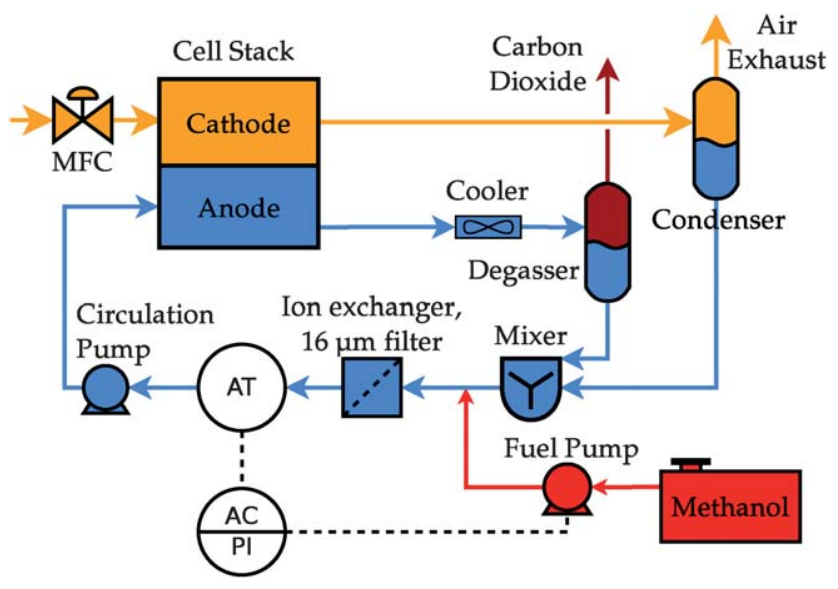

Fig. 2 The flowsheet of the experimental rig. 
All components of the rig are operated by means of a Siemens SIMATIC PCS 7 process control system.

The methanol concentration of solution samples from the mixer tank could be measured by means of an Atago RX-5000 $\alpha$ refractometer.

3.1.1 Fuel-cell stack. The DMFC stack was assembled inhouse and comprises three cells, each with an active area of $26 \mathrm{~cm}^{2}$ ( $65 \mathrm{~mm}$ by $\left.40 \mathrm{~mm}\right)$. The cells were electrically connected in series, whereas the flows were connected in a $\mathrm{Z}$ configuration. ${ }^{7}$ All monopolar plates were built in graphite, and have a singlechannel flow field with cross-section of $1 \mathrm{~mm} \times 1 \mathrm{~mm}$ and a foot width of $0.95 \mathrm{~mm}$, resulting in 20 meanders.

The membrane-electrode assemblies (MEAs) were acquired from Johnson Matthey (type MEA 0281) and use Nafion N115 membranes; they were conditioned according to the procedures specified by the producer.

In the anodic monopolar plate of the central cell a hole was drilled, $31.5 \mathrm{~mm}$ deep and $1 \mathrm{~mm}$ wide, so that a K-type thermocouple could be fitted in it. Preliminary tests confirmed that the temperature in each cell did not deviate measurably from the value measured by the thermocouple in the central cell at any temperature of interest.

The stack's temperature was controlled by a PID controller, tuned with Ziegler-Nichols' rules, which manipulated two Minco HR 5417R heating foils, each with a resistance of $5.3 \Omega$, glued to each end plate of the stack. The temperature measurement was provided by the aforementioned K-type thermocouple.

The stack was electrically connected to a Höcherl \& Hackl ZS512-4 electronic load that allowed to set its current. To verify the voltage of each cell, which could in some conditions be quite different, a Fluke 79 III multimeter was used on a regular basis; as a precaution to safeguard catalyst integrity, it was decided not to run the stack when any cell's voltage fell below $200 \mathrm{mV}$.

3.1.2 Anodic loop. The anodic loop's mixer tank is realised as a glass container with a volume of $500 \mathrm{~cm}^{3}$, in which a stir bar is operated by means of a magnetic stirrer at a constant setting. On

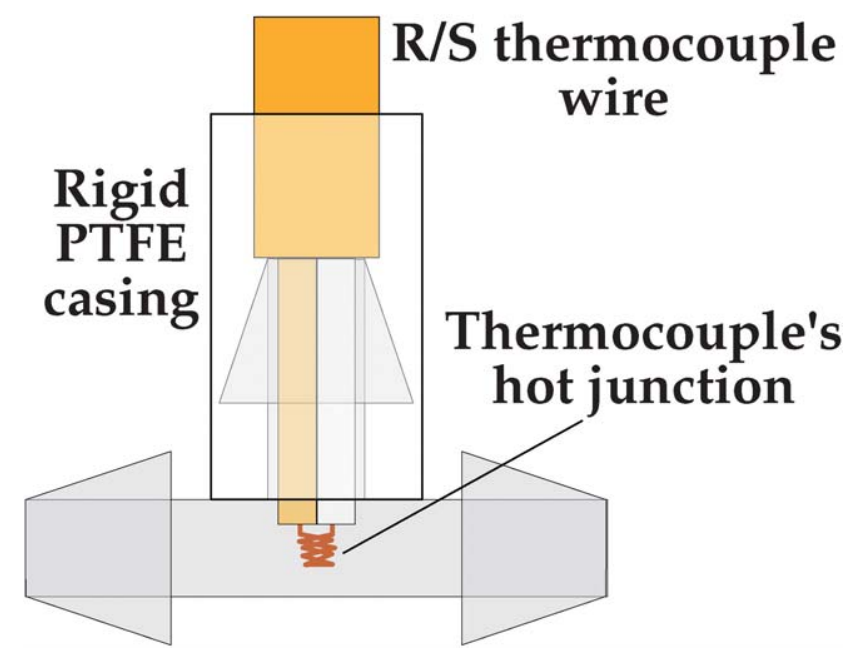

Fig. 3 On-flow R/S thermocouple used in the experimental layout. one side of the mixer, a SensorTechnics BSDX0100D4R pressure sensor is present to infer the solution level in it.

Methanol is pumped from its reservoir tank with an Ismatec Reglo Digital pump using a Tygon 3350 peristaltic tubing with an internal diameter of $0.51 \mathrm{~mm}$, allowing continuous flows as low as $25 \mathrm{~mm}^{3} / \mathrm{min}$. The system's process control system allowed to set lower rates by means of discontinuous flow.

The methanol flow is joined to the main solution flow by means of an in-line mixer. This approach was chosen to produce rapidly a solution of the desired concentration to be fed to the stack, and thereby reduce the time required by experimental runs.

Because of the significant difference in flow rates of solution and make-up methanol, and because of the laminar nature of the two flows, it is not possible to use a simple $\mathrm{T}$ connector to mix them: imperfect mixing would lead to large oscillations in the concentration entering the stack, as noticed in early tests.

Instead, it has been necessary to inject the methanol flow in the middle of the solution flow by adapting a standard $\mathrm{T}$ connector to serve as an in-line mixer, similarly to the cases presented by Sinnot, ${ }^{12}$ as shown in Fig. 4. In the in-line mixer, the main solution flow enters from the side port of a $\mathrm{T}$ connector with internal diameter of $2 \mathrm{~mm}$, and envelops the outlet of a PTFE tube with external diameter $1.6 \mathrm{~mm}$, out of which pure methanol enters the solution flow. The methanol port of the T connector is sealed with PTFE tape. The combined streams exit from the remaining port of the $\mathrm{T}$ connector.

The enriched solution passes then through a cation exchanger to remove any traces of metallic ions that may be present in the flow. The exchanger material is Amberlyst 15. The outlet of the ion exchanger is passed through a ROBU VitraPOR P16 glass filter, with pore size less than $16 \mu \mathrm{m}$, to ensure that no solid particles enter the following concentration sensor.

The methanol concentration sensor is an ISSYS FC6. This sensor is used by a PI feedback controller, tuned with ZieglerNichols' rules, to set the methanol pump's feed rate with the objective of maintaining a certain value of concentration.

The anodic-loop flow is pumped by an Ismatec MCP Standard pump with a Easy-Load II pumping head and a tubing with an internal diameter of $3.2 \mathrm{~mm}$, after which the flow enters the fuelcell stack.

The solution leaving the stack was cooled in a custom-built heat exchanger provided by Institut für Mikrotechnik Mainz, with nominal heat-exchange area $230 \mathrm{~cm}^{2}$ and heat transfer coefficient $188 \mathrm{~W} / \mathrm{m}^{2} \mathrm{~K}$. The exchanger's coolant is dry air, whose

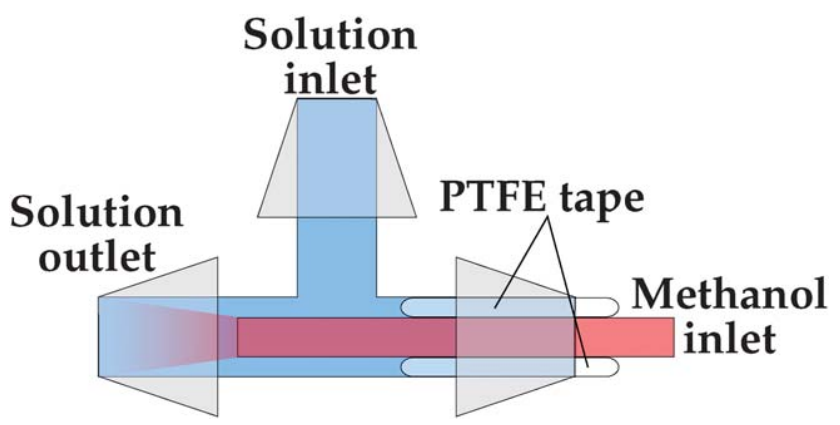

Fig. 4 The in-line mixer used in the experimental setup to enrich the methanol solution before it enters the fuel-cell stack. 
flow is controlled by a Sierra Smart-Trak M100 mass flow controller.

The solution is then separated from the $\mathrm{CO}_{2}$ produced in the stack in a simple, thermally insulated glass separator, and its liquid outlet is returned to the main mixer tank.

3.1.3 Cathodic line. The air entering the cathode can be assumed to be completely dry, and its inflow is controlled by a Sierra Smart-Trak C100 mass-flow controller.

The cathodic outlet enters a custom-built condenser unit provided by Institut für Mikrotechnik Mainz, with nominal heatexchange area $346 \mathrm{~cm}^{2}$ and heat transfer coefficient $82 \mathrm{~W} / \mathrm{m}^{2} \mathrm{~K}$. The condenser's coolant is dry air, whose flow is controlled by a Sierra Smart-Trak M100 mass flow controller.

The water recovered in the condenser is then returned to the anodic loop's mixer.

\subsection{Measurement procedure}

Once the system is properly connected, experimental runs are performed with various values for stack temperature, methanol concentration and current.

Stack temperature is maintained constant by the PCS system's PID temperature controller, and the inlet concentration by its PI concentration controller. Current is then changed from $0 \mathrm{~A}$, in steps of $1 \mathrm{~A}$, to the maximum value that is able to maintain the voltage of all cells above $200 \mathrm{mV}$, which is in general different for various conditions of temperature and concentration.

Experimental data were then logged and monitored. The experimental run was deemed to be over when the methanol pump reached a steady state and maintained that for about half an hour. Depending on concentration and current, transients took typically between 30 minutes and one hour.

The anodic $\lambda$ was set to 10 in all runs; this high value ensures that the concentration through the stack is roughly uniform. As it was unknown at the beginning of the experiment what the values of parameters $k_{m}$ and $k_{x}$ were (indeed, their determination is the objective of the experiment), values obtained from initial tests were used: $k_{m}=8 \mu \mathrm{m} / \mathrm{s}$ and $k_{x}=2 \mu \mathrm{m} / \mathrm{s}$.

To check that $\lambda=10$ was sufficient to maintain a roughly constant concentration through the stack, after every sample point the concentration in the mixer tank was measured with the refractometer, to confirm that the exiting solution had about the same concentration as the entering one; this condition has always been fulfilled in all experimental runs.

The degasser temperature was set to be maintained at $40{ }^{\circ} \mathrm{C}$, in order to minimise the methanol loss from the degasser. The degasser temperature was nevertheless logged and its value included in the calculation of methanol loss, even if it is a negligible term.

The procedure was run for stack temperatures of $50{ }^{\circ} \mathrm{C}, 60^{\circ} \mathrm{C}$ and $70{ }^{\circ} \mathrm{C}$, and for inlet concentrations of $0.7 \mathrm{M}, 1.0 \mathrm{M}$ and $1.5 \mathrm{M}$.

\subsection{Error sources}

The pressure measurement with which the amount of solution in the mixer tank is inferred is sensitive to mixer temperature: with higher temperatures, the solution has lower viscosity, which in turn gives less resistance to the magnetic stir bar. This results in an unreliable measurement until the initial temperature transient has settled, typically 30 minutes after the start of the experimental run.

The FC6 concentration sensor is very sensitive to a series of potential disturbances, such as electrostatic discharges and even small mechanical perturbations. As it is very close to a peristaltic pump, its measurement can be noisy, in particular at high flow rates; this noise is typically in the range of $\pm 0.05 \mathrm{M}$.

The temperature measurements of R/S thermocouples is also quite noisy, because of the small voltage they generate for temperatures in the range of interest for this system. However, stack temperature, which is the main temperature of interest for this study, is measured with a more precise K-type thermocouple $( \pm 0.5 \mathrm{~K})$.

Finally, other sources of error can be a not completely attained steady state or imprecision in the pump setting, which is claimed by the manufacturer to be within $1 \%$ of measurement.

\subsection{Validation of feedforward control}

To validate the results of the parameter regression, the system is run on feedforward methanol control by disconnecting the PI concentration controller in Fig. 2 and letting the neat methanol feed into the mixer vessel instead of the on-line mixer. The flow of neat methanol will be determined by the previously proposed feedforward control law, ${ }^{18}$ using the regressed parameters to calculate control parameters $\hat{a}$ and $\hat{b}$.

\section{Results}

The measured dependence of methanol consumption on current, concentration and temperature is presented in Fig. 5. There, also the reaction consumption given by the current is plotted; data points below this line indicate the presence of some experimental error.

These results are used to perform nonlinear regression on the models introduced in section 2.2, using Matlab's nlinfit function.

The case of constant mass-transport coefficients $(E=0 \mathrm{~kJ} / \mathrm{mol})$ results in the parameters given in Table 1; for the case of coefficients varying according to the activation energy reported by Kauranen and Skou ( $E=11.6 \mathrm{~kJ} / \mathrm{mol})$, in Table 2; for the case of activation energy $E$ as a parameter to estimate, in Table 3 . The case with separate activation energies $E_{m}$ and $E_{x}$ is not reported, as the algorithm was unable to converge to a solution because the parameters are too correlated. Each table reports the estimated values of parameters, their standard deviation, the correlation matrix $C$ and the coefficient of determination $R^{2}$; the correlation matrix is calculated from the covariance matrix returned by nlinfit and the coefficient of determination is calculated from the regression residuals.

The residuals for the three models are plotted in Fig. 6 against current, in Fig. 7 against concentration, and in Fig. 8 against stack temperature.

The system was then run on concentration feedforward control for over 100 hours, with an anodic $\lambda$ of 5 ; the logged inlet concentration is reported in Fig. 9. The test was run with a concentration set point of $1 \mathrm{M}$ and stack temperature of $60{ }^{\circ} \mathrm{C}$. Unfortunately, difficulties with the solution-level sensor caused 


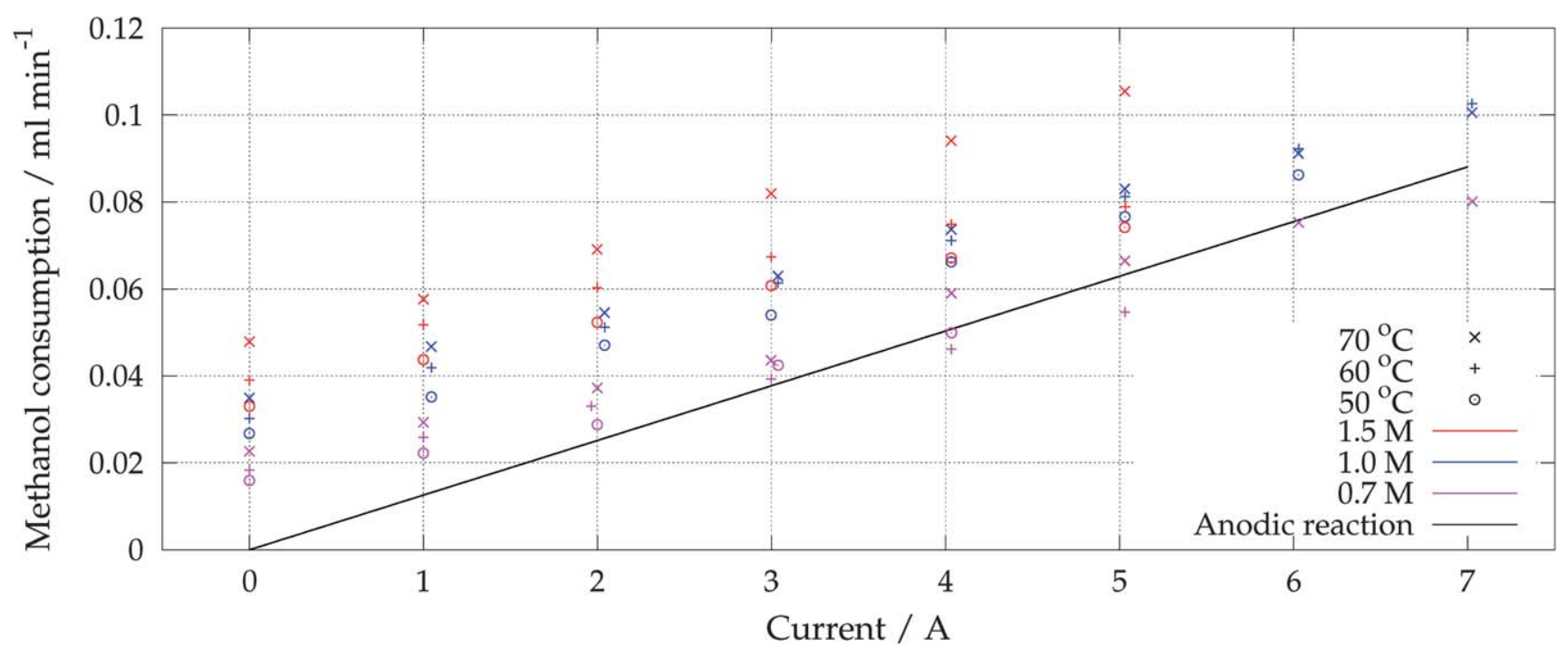

Fig. 5 The experimental dependence of methanol consumption on current for the three values of anodic concentration and the three values of stack temperatures that were considered. The anodic reaction should be an absolute minimum value, and values lower than that indicate experimental error.

Table 1 Results of nonlinear regression for the case of constant mass-transport coefficients

\begin{tabular}{ll}
\hline$k_{m}$ & $(5.6 \pm 0.52) \mu \mathrm{m} / \mathrm{s}$ \\
$k_{x}$ & $(2.0 \pm 0.17) \mu \mathrm{m} / \mathrm{s}$ \\
$C$ & {$\left[\begin{array}{cc}1 & -0.770 \\
-0.770 & 1\end{array}\right]$} \\
& \\
$R^{2}$ & 0.904 \\
\hline
\end{tabular}

Table 2 Results of nonlinear regression for the case of mass-transport coefficients varying with temperature as given by Kauranen and Skou ${ }^{8}$

\begin{tabular}{ll}
\hline$k_{m, 0}$ & $(387 \pm 24.5) \mu \mathrm{m} / \mathrm{s}$ \\
$k_{x, 0}$ & $(150 \pm 9.6) \mu \mathrm{m} / \mathrm{s}$ \\
$C$ & {$\left[\begin{array}{cc}1 & -0.767 \\
-0.767 & 1\end{array}\right]$} \\
& \\
$R^{2}$ & 0.946 \\
\hline
\end{tabular}

Table 3 Results of nonlinear regression for the case of mass-transport coefficients varying with temperature according to activation energy $E$

\begin{tabular}{ll}
\hline$k_{m, 0}$ & $(4100 \pm 3600) \mu \mathrm{m} / \mathrm{s}$ \\
$k_{x, 0}$ & $(1600 \pm 1400) \mu \mathrm{m} / \mathrm{s}$ \\
$E$ & $(18 \pm 2.4) \mathrm{kJ} / \mathrm{mol}$ \\
$C$ & {$\left[\begin{array}{ccc}1 & 0.992 & 0.998 \\
0.992 & 1 & 0.998 \\
0.998 & 0.998 & 1\end{array}\right]$} \\
& \\
& \\
$R^{2}$ & 0.952 \\
\hline
\end{tabular}

the mixer level to oscillate widely at times, inducing dilution or concentration effects.

\section{Discussion}

The results indicate that the most appropriate model is the one that accounts for temperature variation assuming that the activation energy in the Arrhenius expression (19) is set to the value measured by Kauranen and Skou.

The model with constant mass-transport coefficients exhibits less relative precision in the estimation of parameters $k_{m}$ and $k_{x}$, and has a noticeably lower value for $R^{2}$. Furthermore, its residuals show a clear increasing trend when plotted against stack temperature in Fig. 8, pointing to a non-negligible degree of temperature dependence.

On the other hand, the model that tries to obtain an estimate on $E$ as well fails because the precision with which the parameters are known is poor. The correlation matrix has very high values, pointing at too high an interaction between the three parameters. Finally, the residual plots and the $R^{2}$ value for the more complex model are not significantly better than for the model with fixed $E$.

The model with independent activation energies $E_{m}$ and $E_{x}$ has so high correlation between the parameters that the algorithm was unable to converge.

As the data covers most of the area of interest for direct methanol fuel cells, it can be inferred that estimating $E$ is unnecessary, and fixed literature data should be used instead.

The values obtained from regression for $k_{x}$ can be converted to a diffusion coefficient by assuming a membrane thickness of 142 $\mu \mathrm{m}$, obtained by multiplying the nominal thickness of N115 membranes $(127 \mu \mathrm{m})$ with an expansion factor of $12 \%$ due to water soaking, as indicated by their industrial specification. ${ }^{4}$ The temperature-dependent profile obtained in our regression is then compared to literature values in Fig. 10.

This article's estimate for the diffusion coefficient in Nafion membranes is significantly lower than most values reported in the literature. As the purpose of this article is not estimation of this diffusion coefficient, but rather of the methanol consumption, it may be that other unmodelled phenomena (such as the presence 

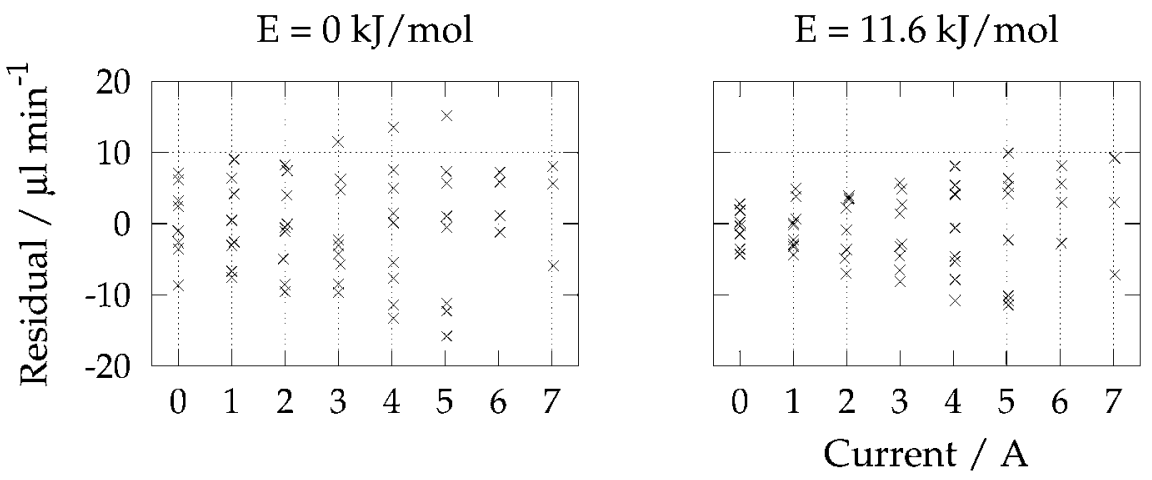

Estimated E

Fig. 6 The residuals of the three models plotted against stack current.
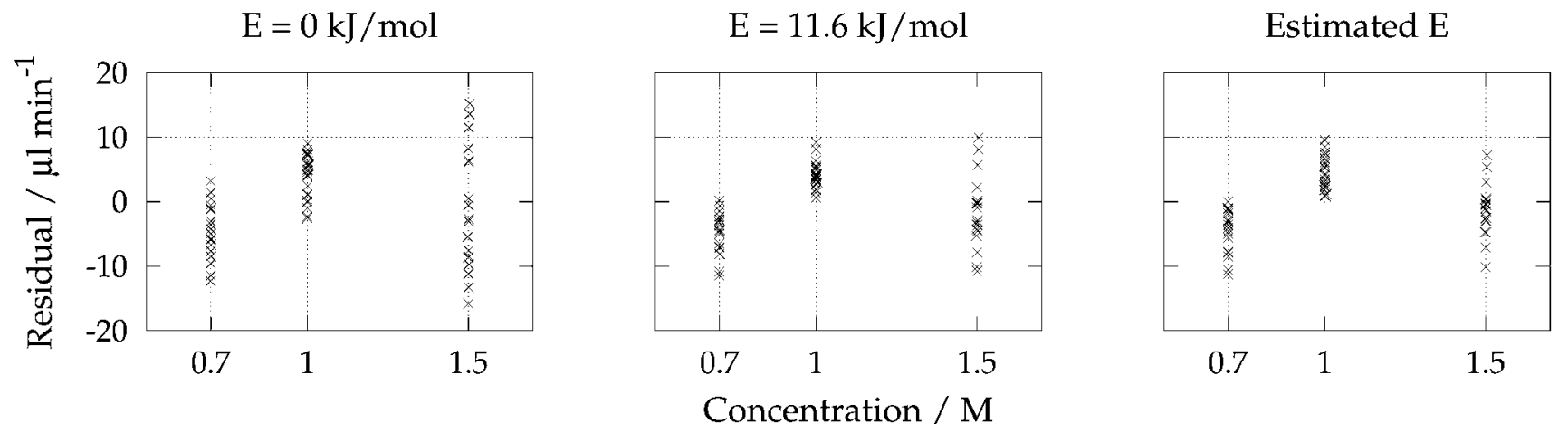

Fig. 7 The residuals of the three models plotted against anodic methanol concentration.
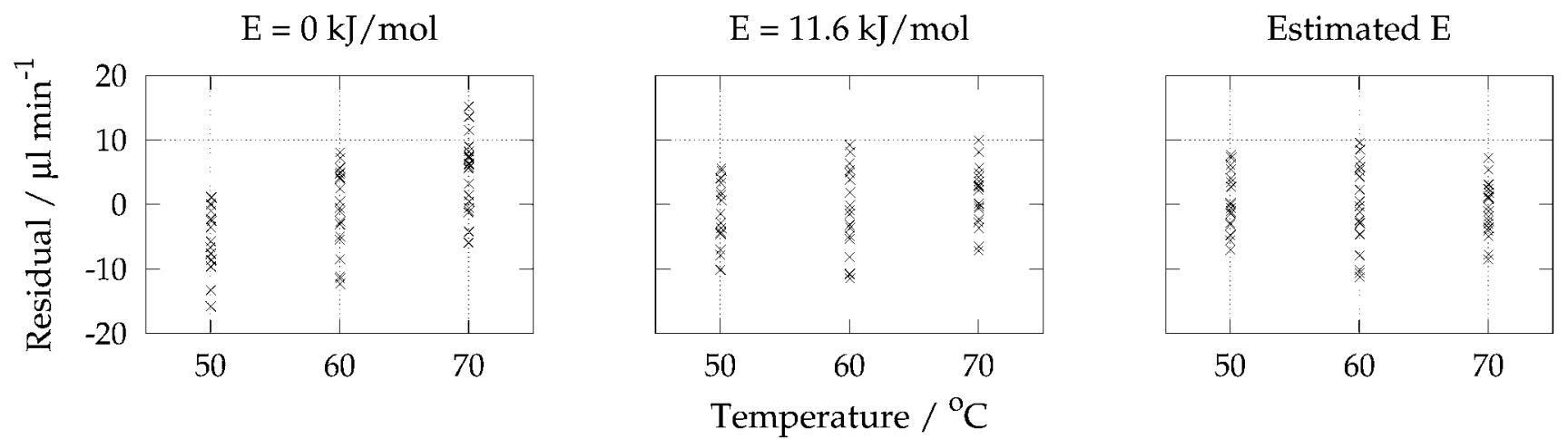

Fig. 8 The residuals of the three models plotted against stack temperature.

of a micro-porous layer) produce an apparently lower value for the diffusion coefficient.

The residuals of all models, when plotted against concentration, have higher values for $1 \mathrm{M}$ than for the other two concentrations (see Fig. 7). This may be an indication of nonlinearity in the mass-transport phenomena, and should not be surprising considering the degree of simplification that this article's mass-transport model introduced.

The expected standard deviation of the feedforwardcontrolled concentration can now be calculated according to eqn (10). Using the extreme values found for the various terms in eqns (16), (17) and (18), the values for $\hat{k}_{m}$ and $\hat{k}_{x}$ and their standard deviations can be substituted as a function of temperature using the Arrhenius relationship (19).

However, due to the structure of the model, the temperature dependence cancels out, and it is possible to provide a general guarantee for the standard deviation of the steady-state concentration value:

$$
\frac{\sigma_{c}}{\bar{c}} \leq 7.83 \%
$$

In the test run of Fig. 9, the concentration set point refers to the average concentration through the cells; since the value of anodic $\lambda$ is no longer so high that it can be assumed that inlet and 


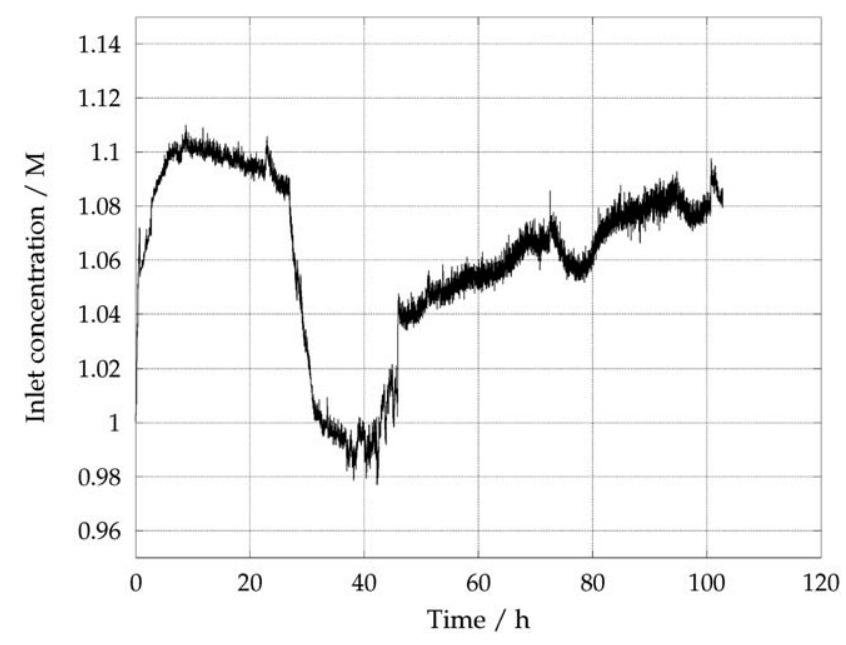

Fig. 9 The inlet concentration during a test run to validate the feedforward control algorithm at $60{ }^{\circ} \mathrm{C}$ and $1 \mathrm{M}$ target average concentration. Concentration was sampled every $4 \mathrm{~s}$, and the measurement has been filtered of sensor noise using a moving average spanning a minute.

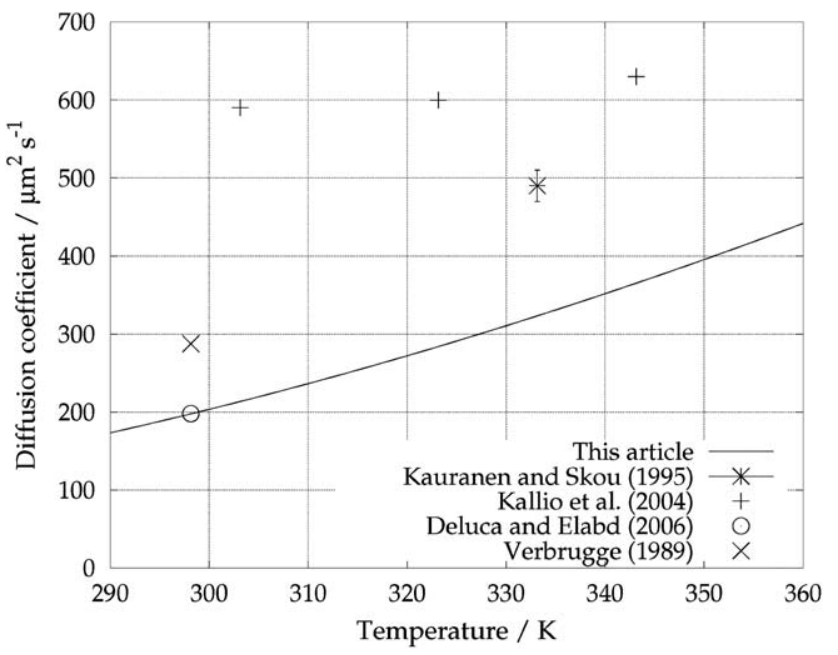

Fig. 10 This article's estimated diffusion coefficient of methanol through the Nafion membrane. Note that Kauranen and Skou and Deluca and Elabd worked with the thicker N117 membrane, and Verbrugge used a $211 \mu \mathrm{m}$ thick membrane. Only Kauranen and Skou provided an error estimate.

outlet concentration are equal, it is expected that outlet concentration will be measurably lower than the inlet. Since the measurement is placed on the inlet, it is therefore to be expected that the measurement report a value slightly higher than $1 \mathrm{M}$, which is the case in the test run. Notwithstanding the disturbances to the test run, feedforward control was able to maintain concentration within the bounds to be expected from eqn (20). It would be advantageous to use a smaller mixer unit, to accelerate the transients and allow more prompt validation runs.

\section{Conclusions}

This article proposed several mathematical models to estimate the cross-over in a direct methanol fuel cell. The selected model assumes two mass-transport coefficients, one from the anodic bulk to the anodic catalyst layer, and another from the anodic catalyst layer through the membrane; these are assumed to vary with temperature with an Arrhenius expression and with an activation energy as given by Kauranen and Skou.

The selected model can be employed in feedforward concentration control, as previously proposed by the authors, and, with the precision with which the model was fit, it was determined that the standard deviation of the tracking error would be less than $8 \%$, which is an acceptable value for control of anodic methanol concentration in DMFCs.

The residuals of the regression analysis suggest that the model is not representing well the dependence on concentration. This is expected, as the model is admittedly a simplification of a much more complex set of phenomena.

A test run to validate feedforward concentration control produced encouraging results; before further tests are run, however, a better sensor for the solution level in the mixer must be implemented.

\section{Nomenclature}

\subsection{Latin symbols}

\begin{tabular}{ll}
\hline$A$ & Cell active area $\left(\mathrm{m}^{2}\right)$ \\
$a$ & Cell parameter $(\mathrm{m} / \mathrm{s})$ \\
$b$ & Cell parameter $(-)$ \\
$c$ & Methanol concentration $\left(\mathrm{mol} / \mathrm{m}^{3}\right)$ \\
$C$ & Correlation matrix $(-)$ \\
$C o v$ & Covariance \\
$D$ & Diffusion coefficient $\left(\mathrm{m}^{2} / \mathrm{s}\right)$ \\
$E$ & Activation energy $\left(\mathrm{J} / \mathrm{mol}^{3}\right)$ \\
$F$ & Faraday constant $(96485 \mathrm{C} / \mathrm{mol})$ \\
$I$ & Current $($ A) \\
$i$ & Current density $\left(\mathrm{A} / \mathrm{m}^{2}\right)$ \\
$k$ & Mass-transport coefficient $(\mathrm{m} / \mathrm{s})$ \\
$N$ & Molar flux $\left(\right.$ mol $\left./ \mathrm{m}^{2} \mathrm{~s}\right)$ \\
$n$ & Amount of substance $(\mathrm{mol})$ \\
$R$ & Ideal gas constant $(8.314 \mathrm{~J} / \mathrm{molK})$ \\
$R^{2}$ & Coefficient of determination $(-)$ \\
$T$ & Temperature $(\mathrm{K})$ \\
\hline
\end{tabular}

\subsection{Greek symbols}

$\begin{array}{ll}\lambda & \text { Reactant excess ratio (-) } \\ \sigma & \text { Standard deviation }\end{array}$

\subsection{Subscripts}

\begin{tabular}{ll}
\hline$c l$ & Catalyst layer \\
$m$ & Mass transport from anode bulk to catalyst \\
$r$ & layer \\
$x$ & Anodic reaction \\
\hline
\end{tabular}




\subsection{Accents}

\begin{tabular}{ll}
\hline$\dot{\mathrm{o}}$ & Flow $\left(\mathrm{s}^{-1}\right)$ \\
$\hat{\mathrm{o}}$ & Estimate \\
$\overline{\mathrm{o}}$ & Set point
\end{tabular}

\section{Acknowledgements}

The authors would like to thank the personnel of the electronic and mechanical workshops of the Max Planck Institute of Magdeburg for their support in the realisation of the experimental rig, and students Neeta Dixit and Marius Kühne for their laboratory work.

\section{References}

1 J. Cruickshank and K. Scott, The degree and effect of methanol crossover in the direct methanol fuel cell, J. Power Sources, 1998, 70(1), 40-47.

2 N. W. Deluca and Y. A. Elabd, Polymer electrolyte membranes for the direct methanol fuel cell: A review, J. Polym. Sci., Part B: Polym. Phys., 2006, 44(16), 2201-2225.

$3 \mathrm{H}$. Dohle, J. Mergel and D. Stolten, Heat and power management of a direct-methanol-fuel-cell (DMFC) system, J. Power Sources, 2002, 111, 268-282.

4 DuPont Fuel Cells, 2009. DuPont Nafion PFSA Membranes. URL http://www2.dupont.com/FuelCells/en_US/assets/downloads/dfc101.pdf.

5 T. J. Ha, J.-H. Kim, H.-I. Joh, S.-K. Kim, G.-Y. Moon, T.-H. Lim, C. Han and H. Y. Ha, Sensor-less control of methanol concentration based on estimation of methanol consumption rates for direct methanol fuel cell systems, Int. J. Hydrogen Energy, 2008, 33(23), 7163-7171.

6 T. Kallio, K. Kisko, K. Kontturi, R. Serimaa, F. Sundholm and G. Sundholm, Relationship between methanol permeability and structure of different radiation-grafted membranes, Fuel Cells: From Fundamentals to Systems, Dec. 2004, 4(4), 328-336.

7 G. Karimi, J. J. Baschuk and X. Li, Performance analysis and optimization of PEM fuel cell stacks using flow network approach, J. Power Sources, 2005, 147, 162-177.

8 P. S. Kauranen and E. Skou, Methanol permeability in perfluorosulfonate proton exchange membranes at elevated temperatures, J. Appl. Electrochem., Sep. 1995, 26(9), 909-917.

9 National Oceanic and Atmospheric Administration, 2008. Global measured extremes of temperature and precipitation. URL http:// www.ncdc.noaa.gov/oa/climate/globalextremes.html.

10 R. H. Perry, D. W. Green, 1997. Perry's Chemical Engineers' Handbook. 7th Edition.

11 T. Schultz and K. Sundmacher, Rigorous dynamic model of a direct methanol fuel cell based on maxwell-stefan mass transport equations and a flory-huggins activity model: Formulation and experimental validation, J. Power Sources, 2005, 145, 435-462.

12 R. K. Sinnot, 1999. Chemical Engineering Design, 3rd Edition. Vol. 6 of Coulson \& Richardson's Chemical Engineering. ButterworthHeinemann.

13 K. Sundmacher, T. Schultz, S. Zhou, K. Scott, M. Ginkel and E. D. Gilles, Dynamics of the direct methanol fuel cell (DMFC): experiments and model-based analysis, Chem. Eng. Sci., 2001, 56, 333-341.

14 J.-M. Tarascon and M. Armand, Issues and challenges facing rechargeable lithium batteries, Nature, 2001, 414(6861), 359-367.

$15 \mathrm{M}$. W. Verbrugge, Methanol diffusion in perfluorinated ion-exchange membranes, J. Electrochem. Soc., 1989, 136(2), 417-423.

16 S. von Andrian and J. Meusinger, Process analysis of a liquid-feed direct methanol fuel cell system, J. Power Sources, 2000, 91, 193-201.

17 C.-Y. Wang, Fundamental models for fuel cell engineering, Chem. Rev., 2004, 104(10), 4727-4765.

$18 \mathrm{~F}$. Zenith and U. Krewer, Modelling, dynamics and control of a portable DMFC system, J. Process Control, 2010, 20(5), 630-642.

19 H. Zhao, J. Shen, J. Zhang, H. Wang, D. P. Wilkinson and C. E. Gu, Liquid methanol concentration sensors for direct methanol fuel cells, J. Power Sources, 2006, 159(1), 626-636.

20 T. S. Zhao, C. Xu, R. Chen and W. W. Yang, Mass transport phenomena in direct methanol fuel cells, Prog. Energy Combust. Sci., 2009, 35(3), 275-292. 